\title{
Josef Zechner zum 65. Geburtstag
}

\author{
Stefan Bogner $\cdot$ Stefan Pichler
}

Online publiziert: 16. Juni 2020

(C) Schmalenbach-Gesellschaft für Betriebswirtschaft e.V. 2020

Am 2. Juli 2020 feierte Josef Zechner seinen fünfundsechzigsten Geburtstag.

Josef Zechner schloss das Diplomstudium aus Betriebswirtschaftslehre mit den Spezialisierungen Finanzwirtschaft und Rechnungswesen an der Karl-Franzens-Universität Graz 1978 ab. 1980 promivierte er ebendort zum Doktor der Sozial- und Wirtschaftswissenschaften mit der Arbeit „Optimale Erweiterungs- und Ersatzzeitpunkte von Anlagen in wachsenden Unternehmungen“. Seine Fokussierung auf finanzwirtschaftliche Forschung manifestierte sich mit der Habilitationsschrift „,Der Einfluss von Steuern auf die optimale Kapitalstruktur" und der Verleihung der Venia Legendi durch die Karl-Franzens-Universität Graz 1987. 2007 erhielt Josef Zechner für seine richtungsweisenden Beiträge zur Weiterentwicklung der Bank- und Finanzierungstheorie die Ehrendoktorwürde der Fakultät für Betriebswirtschaftslehre der Ludwig-Maximilians-Universität München.

1985 beendete Josef Zechner sein Beschäftigungsverhältnis als Universitätsassistent an der Karl-Franzens-Universität Graz um an der University of British Columbia, Vancouver, Canada eine Stelle als Assistant Professor anzunehmen, an der er bereits 1982/83 einen Forschungsaufenthalt als Visiting Scholar verbracht hatte und 1990 zum Associate Professor with tenure aufstieg. Im akademischen Jahr 1991/92 war er zudem Research Scholar an der Graduate School of Business, Stanford University, USA.

Mit der Annahme des Rufes an die Universität Wien kehrte Josef Zechner 1993 nach Österreich zurück. Sein gemeinsames Wirken mit Engelbert Dockner für 15 Jahre an der Universität Wien war hauptverantwortlich für die Etablierung Wiens als einen der führenden finanzwirtschaftlichen Forschungsstandorte in Europa. Diese Rolle verstärkte sich noch 2008 durch den gemeinsamen Wechsel mit

\footnotetext{
S. Pichler $(\square)$

Department of Finance, Accounting and Statistics, WU Wien, Welthandelsplatz 1, A-1020 Wien, Österreich

E-Mail: Stefan.Pichler@wu.ac.at
} 
Engelbert Dockner an die Wirtschaftsuniversität Wien (WU), wo Josef Zechner bis heute als Professor of Finance and Investments wirkt.

Josef Zechner ist Sprecher des PhD Programms Vienna Graduate School of Finance (VGSF), das er als gemeinsame Initiative mit dem Institut für Höhere Studien, der Universität Wien und der WU 2005 mitbegründete. Er ist wirkliches Mitglied der Österreichischen Akademie der Wissenschaften und Research Fellow of the Centre for Economic Policy Research (CEPR). Von 2003 bis 2012 war er Managing Editor der Review of Finance (RoF). Er war ebenfalls Präsident der European Finance Association (EFA), der German Finance Association (DGF) und der Western Finance Association (WFA) und er ist gegenwärtig Vizepräsident der Society for Financial Studies (SFS). Josef Zechner war Mitglied des Advisory Scientific Committee des European Systemic Risk Board (ESRB) der European Central Bank und ist Fellow der Finance Theory Group.

Diese bei weiten nicht vollständige Aufzählung seiner Funktionen und Mitgliedschaften in internationalen Berufs- und Fachvereinigungen belegen seine herausragende weltweite Stellung in der finanzwirtschaftlichen Forschung und Praxis. So ist Josef Zechner noch immer der einzige, nicht aktiv an einer nordamerikanischen Universität lehrende Präsident in der mehr als 50-jährigen Geschichte der WFA. Sein bedeutender Beitrag zur finanzwirtschaftlichen Forschung wird nicht nur durch seine eigenen Arbeiten, sondern auch durch seine Herausgeberschaften eindrucksvoll belegt. So etablierte sich die RoF gerade in seinem fast 10 Jahre dauernden Wirken als eine der führenden Fachzeitschriften. Ebenso beispielhaft war und ist seine Rolle als Förderer des wissenschaftlichen Nachwuchses, als Kollege und als Freund. Seine menschliche Wärme und Herzlichkeit werden von allen bestätigt, die das Glück hatten und noch haben, mit ihm zusammenarbeiten zu dürfen.

Josef Zechners internationale Bedeutung für die Entwicklung des Fachs Finanzwirtschaft ist sehr schwer in einem Beitrag mit begrenzter Zeichenzahl zu fassen. Seine erste A-Publikation erschien vor 32 Jahren im Journal of Finance (gemeinsam mit Michael Brennan und Vojislav Maksimovic zu Vendor Financing) und er publiziert auch weiterhin auf diesem höchsten Niveau, in den letzten Jahren sogar mit zunehmender Frequenz (zuletzt gemeinsam mit Paul Schneider und Christian Wagner zu Low Risk Anomalies). Besonders beeindruckend ist aber der Umstand, dass es Josef Zechner auf nahezu einzigartige Weise gelungen ist, in mehreren, thematisch eigentlich oft weit auseinanderliegenden Teilgebieten bahnbrechende Erkenntnisse zu erzielen, manche Teilgebiete sogar zu begründen.

Ohne Anspruch auf Vollständigkeit seien hier nur ein paar Beispiele ausgewählt. Die 1989 im Journal of Finance erschienene Arbeit mit Edwin Fischer und Rob Heinkel über die Wahl der optimalen dynamischen Kapitalstruktur gilt als erste Arbeit in diesem Gebiet und als eine der wenigen großen Entwicklungsschritte in der Kapitalstrukturtheorie. Die 2010 im Journal of Finance erschienene Arbeit mit Jonathan Berk und Richard Stanton zum Zusammenhang von Humankapital, Insolvenzrisiko und Kapitalstrukturentscheidungen gilt als eine der ersten Arbeiten zu „Labour Finance“. Auch die neueren Entwicklungen in der Forschung zum Thema Cross Listing (2001 mit Marco Pagano, Otto Randl und Alisa Roell) und Large Shareholder Activism (1994 mit Anat Admati und Paul Pfleiderer) gehen auf Arbeiten von Josef Zechner zurück. Ganz besonders aktuell erscheint der Umstand, 
dass auch im heute immer bedeutender werdenden Feld des Impact Investings Josef Zechner eine der ersten Arbeiten dazu verfasst hat (bereits 2001 mit Rob Heinkel und Alan Kraus).

In den ersten Jahren seines Wirkens hat sich seine Forschung eher im Bereich Corporate Finance bewegt, wobei er oft ein besonderes Augenmerk auf die Rolle der Ausgestaltung des Fremdkapitals legte (so bereits 1991 in einer Arbeit mit Vojislav Maksimovic zu Debt, Agency Costs, and Industry Equilibrium, oder erst 2020 mit Jaewon Choi und Dirk Hackbarth zu Granularity of Corporate Debt). In den letzten Jahren standen immer öfter auch Fragen im Bereich des Asset Managements und der Marktstruktur im Mittelpunkt seines Interesses (so zum Beispiel die 2014 mit Nils Friewald und Christian Wagner verfasste Arbeit über Kreditrisikoprämien und Aktienrenditen oder die 2011 mit Neal Stoughton und Youchang Wu verfasste Arbeit zur Intermediation im Investment Management).

Aufgrund der enormen Breite und des großen Umfangs seines Wirkens ist es nahezu unmöglich, die wissenschaftliche Bedeutung seines Wirkens in einem Satz zusammenzufassen. Am ehesten würde aus unserer Sicht dem die Einsicht gerecht werden, dass Finanzwirtschaft trotz aller Ausdifferenzierungen in Corporate Finance und Asset Pricing nach wie vor auf einem vereinenden Gedankengebäude beruht. Preisbildung auf Wettbewerbsmärkten, Entscheidungen unter Unsicherheit und die durch unterschiedliche Anspruchsinteressen entstehenden Asymmetrien sind auf vielfältige Weise miteinander verbunden und können nur durch eine gemeinsame Betrachtung aller Dimensionen sinnstiftend erklärt werden.

Wir sind überzeugt, dass sich Josef Zechner auch nach diesem Geburtstag nicht in den akademischen Ruhestand begeben wird, sondern vielmehr noch viele Jahre innovative und kreative Forschung auf höchstem Niveau betreiben wird. Wir gratulieren Josef Zechner im Namen seiner Schüler/innen und Kolleg/innen ganz herzlich und wünschen ihm für die Zukunft alles Gute. 